Review

\title{
Comparative Study on Microbial Communities of Soil, Grape Must and Wine Fermentation of Cabernet Sauvignon Inoculated with three Commercial Yeast of Saccharomyces cerevisiae
}

\author{
${ }^{1}$ Rongbin Li, ${ }^{1} \mathrm{Xu}$ Shi, ${ }^{1}$ Yaqiong Liu, ${ }^{1}$ Haoran Wang, ${ }^{2}$ Xiaofang Fu, \\ ${ }^{2}$ Huanxiang Wang, ${ }^{1}$ Jianlou Mu and ${ }^{1}$ Jie Wang \\ ${ }^{1}$ College of Food Science and Technology, Hebei Agricultural University, Hebei, 071000, China \\ ${ }^{2}$ China Great Wall Wine Company Limited, Hebei, 075499, China
}

\author{
Article history \\ Received: 20-07-2021 \\ Revised: 13-11-2021 \\ Accepted: 17-11-2021 \\ Corresponding Author: \\ Jie Wang \\ College of Food Science and \\ Technology, Hebei \\ Agricultural University, Hebei, \\ 071000, China \\ Email: wangjie@hebau.edu.cn
}

\begin{abstract}
This study examined the microbial diversity in soil, grape must and wine fermentation of Cabernet Sauvignon inoculated with three different starters (S01: Xinjiang Saccharomyces cerevisiae CEC01; SCA: Ningxia Saccharomyces cerevisiae CECA; S96: Saccharomyces cerevisiae AWRI 796) using a high-throughput sequencing technique. The results showed that 227 bacterial genera and 20 fungi genera were shared in all samples, consisting of Saccharomyces, Filobasidium, Colletotrichum, Alternaria by Venn diagram analysis. Principle component analysis showed the microbiota structures between S01, SCA and S96 fermentation were similar, the major bacterial genera were Pseudomonas, unclassified_f_Enterobacteriaceae and Lactobacillus, whereas the major fungi genus was Saccharomyces. The biomarkers of bacterial genera in S01, SCA and S96 groups were detected using LEfSe analysis, in which Komagataeibacter, Micromonospora, Streptomyces, Brevibacterium and Agromyces were core microorganisms in the S01 group, SCA fermentation increased the relative abundance of Lactobacillus and Oenococcus, family Ruminococcaceae was dominant in the S96 group. The distinctions in fungi communities between S01, SCA and S96 group were not observed during the fermentation. Understanding of microbial diversity could aid to promote the formation of regional characteristics and the development of high-quality wines through the management of existing microorganisms in future.
\end{abstract}

Keywords: Wine, Microbial Community, Fermentation, Yeast, High-Throughput Sequencing

\section{Introduction}

Cabernet Sauvignon grape, one of the most important Vitis vinifera grape varieties, is originated from the region of Bordeaux, France and introduced to China in 1892 (de Castilhos et al., 2017). The juices and wines produced from Cabernet Sauvignon grapes present high quality and specific features, such as a particular taste, high content phenolic compouds and significant antioxidant activity, therefore they are widespread in the primate wine making contries (Radovanovic et al., 2016). While wines made from the same grape variety that growing in different regions are favored by people because of their unique characteristics, collectively referred to as "terroir".
Shacheng (Hebei Province), located at $40^{\circ}$ north latitude, is one of the golden zones of grape cultivation in the world with characteristics of slightly acidic soil, large temperature difference between daytime and night time, ample light and hot rainy season. The wine production from Cabernet Sauvignon grape in Shacheng has been devolped as one of the representative "terroir" variety in China (Jiang et al., 2013).

It has been well known that microorganisms play an important role in regulating the health and growth of vine and grapes, as well as wine production (Barata et al., 2012). The microbes of grape skin could stem from the vineyard soil, precipitation (rainfall, snow and hail), air and animal transmission (especially bees) (Zarraonaindia et al., 
2015; Lam et al., 2015; Morrison-Whittle et al., 2018). Increasing evidence have supported grape-associated yeasts would participate in wine fermentations affecting the organoleptic characteristics of the wines (Liu et al., 2020), the same process can also be explained in reverse as the transfer of yeast from the winery to the nearby vineyards, thereby affecting the local native yeast community. The persistence of these yeasts in the soil, grapes, vines or processing environment is difficult to determine. Therefore, it is necessary to investigate the growing environment (especially the soil) and the microbial community carried by the grapes to understand the formation of wine flavor characteristics and quality control.

The conversion of grape must into wine is a complicated process involving the participation of many microorganisms and Saccharomyces cerevisiae (S. cerevisiae) is mainly responsible for alcohol fermentation. Traditionally, wine fermentation is spontaneously carried out by indigenous yeasts that appear on the grapes, or yeasts from wine cellars and equipment during the fermentation. In the modern wine industry, inoculation with a single strain of $S$. cerevisiae has been widely used to produce wine with strong stability and consistency (Suzzi et al., 2012) due to its winemaking properties, such as fermentation ability, enhanced wine aroma, low production of hydrogen sulfide, tolerance of temperature, ethanol and pressure. Different strains of $S$. cerevisiae for fermentation of grape must have a very important impact on wine quality, therefore many wine researchers prefer to use and select indigenous $S$. cerevisiae for the wine fermentation (Tzanetakis et al., 2006). For example, based on specific criteria, Nikolaou et al. (2006) have screened indigenous yeast strains from hundreds of isolates for developing unique regional wines. Aponte et al. (2016) have pointed that the indigenous $S$. cerevisiae M3-5 isolated from the "Moscato di Saracena" wine was more suitable for winemaking than commercial strains. Ortiz et al. (2013) have demonstrated that $95 \%$ of isolated yeasts in the spontaneously fermented wines of "La Mancha" region consumed all sugars within 15 days and successfully completed the fermentation. Nine $S$. cerevisiae strains have been evaluated for their ability to reduce the adsorption of tannins on salivary proteins and there were differences between the strains, which has proven that the selection of yeast would be the key to determining changes in color and astringency profile of red wines (Rinaldi et al., 2016).

In addition to $S$. cerevisiae, other bacterial microorganisms in the must release metabolites, which lead to changes in the chemical environment during fermentation and affect the composition and characteristics of wine. Therefore, having more information about dynamic changes of the microbial community during fermentations inoculated with different $S$. cerevisiae is helpful for wine-makers to monitor the alcoholic fermentation, meanwhile modulate the gustative and mouthfeel of red wines.
On a global scale, the wine industry is an important socio-economic activity. The total wine production worldwide is approximate 250 million liters in 2021 (OIV, 2020; Statista, 2020). The worldwide wine industry comprises thousands of distinct geographic regions. For example, nearly 1600 cellar doors in Australia and 212 companies at least in China are spread throughout the geographically classified wine regions, which are marketed collectively according to the unique characteristics of terroir (Lewis et al., 2021). In this study, the high-throughput sequencing was used to analyze microbial community diversity of soil, grape juice and wine producing from Shacheng, Hebei Province, China. Three commercial yeast, Xinjiang $S$. cerevisiae CEC01, Ningxia $S$. cerevisiae CECA and $S$. cerevisiae AWRI 796, were used to perform the Cabernet Sauvignon wine fermentations, aiming to explore the differences in the dynamics of microbial diversity. These results would contribute to the understanding of the relevance between regional microbiome and wine quality and help to discover the dominant microbial genera under different fermentation starter, which would offer valuable analysis for winemakers in monitoring fermentation processes and controlling the quality and flavor of wine in Shacheng.

\section{Materials and Methods}

\section{Sample Collection and Preparation}

The Cabernet Sauvignon vineyard soil samples in the wine grape producing area $\left(\mathrm{N} 40^{\circ} 4^{\prime}, \mathrm{E} 115^{\circ} 52^{\prime}\right)$ were obtained and named as VS sample. In order to get the unbiased VS sample, the five-point sampling method was used and the soil was cut vertically with a sterilized soil shovel at a depth of $20 \mathrm{~cm}$. Approximately $0.5 \mathrm{~kg}$ of soil was sampled at each sampling point and stored in a sterile airtight bag. Soil samples were airdried, gently ground and sieved through a $2 \mathrm{~mm}$ nylon sieve and stored at $-80^{\circ} \mathrm{C}$ until analysis.

Fully mature Cabernet Sauvignon grapes (soluble solids, 23-24 ${ }^{\circ}$ Brix; sugar content, 225.1-232.2 g/L of glucose; $\mathrm{pH}, 3.43-3.45$; total acidity, 5.5-6.3 g/L of tartaric acid) were collected from the Shacheng vineyard in 2019. After the rigorous screening process, Cabernet Sauvignon grapes were destemmed, lightly crushed into grape must and named as GM sample.

A part of group must was cooled to $10 \pm 1{ }^{\circ} \mathrm{C}$ for $24 \mathrm{~h}$ to make the clarification process through natural settlement before alcoholic fermentation. Then, $50 \mathrm{mg} / \mathrm{L}$ of $\mathrm{SO}_{2}$ and $3 \mathrm{~g} / \mathrm{Kg}$ of pectinase (LAFFORT, France) were added to the group must and the loading volume was $80 \%$ of the $90000 \mathrm{~L}$ tank capacity. The fermentation was started by $S$. CEC01, $S$. cerevisiae CECA, $S$. cerevisiae AWRI 796 and namely as S01, SCA and S96, respectively. Among them, S. cerevisiae CEC01 and $S$. cerevisiae CECA were obtained from Angle Yeast Co., 
Ltd (China) and S. cerevisiae AWRI 796 was obtained from Australian Wine Research Institute (Australian). The addition amount of yeast was 20-30 g/100 L. Fermentation was maintained at a controlled temperature $28^{\circ} \mathrm{C}$ for 10 days with pumping three times per day. Samples were collected for analysis at days $0,2,4,6,8$ and 10 during the alcohol fermentation and then the alcoholic fermentations were considered to be finished when the content of total residual sugar content was below $4 \mathrm{~g} / \mathrm{L}$. All samples were stored at $-80^{\circ} \mathrm{C}$ until analysis.

\section{DNA Extraction and PCR Amplification}

The total genomic DNA was extracted from samples using FastDNA® SPIN Kit (MP Biomedicals, USA) according to the manufacturer's instructions. The integrity of DNA was then checked by $1 \%$ agarose gel electrophoresis. Primers 338F/806R and ITS1F/ITS2R were used to amplify the $\mathrm{V} 3-\mathrm{V} 4$ region of bacterial $16 \mathrm{~S}$ rRNA and fungal ITS1 regions for Miseq sequencing, respectively. The TransStart Fastpfu DNA Polymerase was applied in PCR amplification reactions of bacterial 16S rRNA, TaKaRa rTaq DNA Polymerase was used to amplify the ITS1 rDNA regions of fungi. The PCR amplification was carried out in a volume of $20 \mu \mathrm{L}$. The amplified products were visualized by $2 \%$ agarose gel electrophoresis and then purified by the AxyPrep DNA Gel Extraction Kit (AXYGEN Biosciences, Union City, CA, USA).

\section{Illumine Miseq Sequencing and Data Processing}

Both libraries were sequenced on the Illumina Miseq platform by Majorbio Bio-Pharm Technology Co., Ltd. (Shanghai, China). Raw reads obtained from the Illumina platform were filtered to obtain high quality sequences (length $>150 \mathrm{bp}$, the lowest overlap length $<10 \mathrm{bp}$, no read segment containing the base 'N') with the QIIME (ver. 1.8.0). For both bacteria and fungi, non-repetitive sequences were clustered to the Operational Taxonomic Units (OTUs) at a 97\% similarity using RDP Classifier (version 2.2 http://sourceforge.net/projects/rdp-classifier/). Alpha diversities indices used for analyzing the species diversity of samples were calculated by Mothur (ver.1.31.2).

\section{Statistical Analysis}

Significant differences in the means of alpha diversity indices among sample groups were determined by Student's t-test at $p<0.05$. Venn diagrams were performed by an $\mathrm{R}$ package (version 3.3.1) to present unique and shared genera. Principal component analysis was performed to explore the correlation of three $S$. cerevisiae fermentaions samples using an $\mathrm{R}$ package. Differences in the relative abundances of bacteria and fungi genera among grapes must and wine fermentation samples groups were explored using Kruskal-Wallis tests and adjusted by False Discovery Rate (FDR)-corrected p-values. Linear discriminant analysis Effect Size (LEfSe) was conducted using LEfSe package

(http://huttenhower.sph.harvard.edu/galaxy/root?tool_id=lef se_upload).

\section{Results}

\section{Community Alpha Diversity}

After processing to remove low-quality sequences and chimaeras, across all samples, the numbers of Operational Taxonomic Units (OTUs with a threshold of 97\%) were 3129 and 356 for bacteria and fungi, respectively. The alpha diversity indices of soil, grape must and fermented wine samples based on 16S rRNA and ITS sequencing were presented in Table 1 to evaluate the bacterial and fungal diversities and abundances. Good's coverage of all samples reached up to 1.0 , which demonstrated that the sequencing data coverage gave a satisfactory description of the microbial diversity. Overall, based on these results of Shannon, Simpson, ACE and Chao indices, there was a similar tendency for the diversity and richness of micribitia community of bacteria and fungi, that was the VS sample > GM sample > fermented wine samples and the diversity of bacteria was higher than that of fungi. It was clear that the highest alpha diversity indices $(\mathrm{p}<0.05)$ in three yeast strains fermented samples were found at 2 th day, which indicated that the bacterial and fungi community richness at 2 th day was the highest in this stage.

\section{Composition of Microorganism Communities}

The bacterial and fungi communities at the genus level of five sample groups were showed in Fig. 1. The bacterial communities of VS sample were complicated and the relative abundance of each bacteria was lower than other samples. The most abundant bacterial genera in VS was norank_f_JG30-KF-CM45 and followed by Arthrobacter, Streptomyces, Actinobacteria, Nocardioides, Blastococcus, Bacillus, norank_f_Geminicoccaceae and the other genera with relative abundance less than $1 \%$ accounting for $51.06 \%$. The detected bacterial genera across GM and wine samples had a similar tendency, except the higher levels of Sphingomona, norank_f_Mitochondria and Massilia in grape must comparied with the alcohol fermentation samples. After inoculating yeast for fermentation, the Pseudomonas, unclassified_f_Enterobacteriace and Lactobacillus level increased, Sphingomona and norank_f_Mitochondria presented an opposite profile. (Figure 1A). The fungi communities presented a dramatic decrease in microorganism communities' complexity with a few dominant species (Fig. 1B). The dominant genera in VS sample were Fusarium $(23.69 \%)$ and unclassified-cSordariomucetes, unclassified_f_Nectriaceae, unclassifiedo-Hypocreales (53.07\%). For the GM group, Wickerhamomyces, Cladosporium and Schwanniomyces were the dominant genera, representing 65.57, 9.07 and $7.24 \%$ of the total genera, respectively. However, during 
the whole alcohol fermentation period, $98.2 \%$ of organisms belonged to the genera Saccharomyces.

The changes in the bacterial communities during Cabernet Sauvignon wine fermentation were shown in Fig. 2A. Main genera including Pseudomonas, unclassified_f_Enterobacteriaceae, Sphingomona and Lactobacillus were identified during the S01 sample fermentation. It was worth noting that Fructobacillus had the highest abundance value in samples on day $2(22.14 \%)$ and was present in small amounts in the remaining samples. The norank_f_Mitochondria (2.48-3.89\%) was predominant in fermentation 0, 2 days and decreased along with the fermentation of wine. For the SCA sample fermentation, Lactobacillus, Pseudomonas and unclassified_f_Enterobacteriaceae, were the major bacterial communities. Lactobacillus was present at relatively low levels on day 0 and day 2 (6.56 and 2.25\%, respectively) and steadily increased to highest levels on day $6(55.13 \%)$, then it showed a decreasing trend and finally decreased to $18.45 \%$ at the end of fermentation. Similar to S01 fermentation, Fructobacillus had the highest abundance in samples on day $2(50.31 \%)$. For the genus Oenococcus, it gradually increased throughout wine fermentation reaching highest level (about 13\%) on day 8 and day 10 . In the S96 sample fermentation, the main bacterial genera were Pseudomonas and unclassified_f_Enterobacteriaceae. Lactobacillus was no longer a dominant genus compared to that of SCA fermentation, which only appeared in a high level on day 2 $(41.98 \%)$. In Fig. 2B, succession changes of fungi communities were observed during Cabernet Sauvignon wine fermentation. The genus Saccharomyces was extremely abundant (more than 99\%) in all samples.

The shared genera among soil, must and wine fermentation samples were demonstrated in the Venn diagrams (Fig. 3). As shown in Fig. 3A, the VS sample had 461 OTUs, GM sample had 387 OTUs and the fermented samples (S01, SCA and S96) had 719, 678 and 804 OTUs for bacterial, respectively. 227 bacterial genera were found in all samples of the five groups confirming a high consistency in the indigenous microorganism, mainly including Massilia, Cohnella, Sphingomonas, Alkaliphilus, Xylanimonas and Pedobacter. 352 bacterial genera were shared in GM and fermention samples, whereras a higher degree of shared genera shown between S01, SCA and S96 samples were 571 OTUs for the bacterial genera. Fermented samples inoculated with $S$. cerevisiae AWRI 796 were differentiated compared with fermentation samples inoculated with other yeast strains, because they consisted of the following bacterial genera with higher levels: Leptolyngbya_VRUC_135, Cytophaga, Fretibacterium, Desulfobacca, Capnocytophaga and Campylobacter. The fungi genera analysis for each group was shown in Fig. 3B. Specifically, 97, 112, 57, 62 and 73 fungi genera were identified in VS, GM, S01, SCA and S96 samples, respectively. 20 fungi genera were shared in all samples including Saccharomyces, Filobasidium, Colletotrichum, Alternaria, Gibberella, Acremonium, Aspergillus and so on. Compared with GM, 4, 11 and 9 genera were not identified in S01, SCA and S96 samples, respectively. In addition, 38 fungi genera were shared between S01, SCA and S96 fermentation samples.

Table 1: The alpha diversity index of bacterial and fungi in different samples

\begin{tabular}{|c|c|c|c|c|c|c|c|c|c|c|}
\hline \multirow{2}{*}{$\begin{array}{l}\text { Sample } \\
\text { group }\end{array}$} & \multicolumn{2}{|l|}{ Shannon } & \multicolumn{2}{|l|}{ Simpson } & \multicolumn{2}{|l|}{ ACE } & \multicolumn{2}{|l|}{ Chao } & \multicolumn{2}{|c|}{ Goods coverage } \\
\hline & bacterial & fungi & bacterial & fungi & bacterial & fungi & bacterial & fungi & bacterial & fungi \\
\hline VS & 6.29 & 2.73 & 0.00 & 2.73 & 1758.30 & 214.09 & 1754.10 & 211.04 & 1.00 & 1.00 \\
\hline GM & 3.77 & 0.81 & 0.07 & 0.81 & 1088.30 & 197.99 & 926.72 & 194.75 & 1.00 & 1.00 \\
\hline S01_0d & 2.68 & 0.00 & 0.23 & 0.00 & 761.55 & 0.00 & 616.03 & 2.00 & 1.00 & 1.00 \\
\hline S01_2d & 3.53 & 0.08 & 0.10 & 0.08 & 1282.10 & 193.93 & 972.69 & 107.38 & 1.00 & 1.00 \\
\hline S01_4d & 3.45 & 0.01 & 0.14 & 0.01 & 973.72 & 43.31 & 957.03 & 26.33 & 1.00 & 1.00 \\
\hline S01_6d & 2.45 & 0.00 & 0.27 & 0.00 & 860.07 & 0.00 & 821.36 & 4.00 & 1.00 & 1.00 \\
\hline S01_8d & 2.33 & 0.00 & 0.28 & 0.00 & 867.79 & 0.00 & 885.56 & 7.00 & 1.00 & 1.00 \\
\hline S01_10d & 2.58 & 0.01 & 0.23 & 0.01 & 750.48 & 81.08 & 754.58 & 27.00 & 1.00 & 1.00 \\
\hline SCA_0d & 3.52 & 0.00 & 0.10 & 1.00 & 1102.90 & 22.00 & 890.33 & 12.00 & 1.00 & 1.00 \\
\hline SCA_2d & 2.68 & 0.14 & 0.27 & 0.96 & 1444.80 & 126.30 & 1029.10 & 94.08 & 1.00 & 1.00 \\
\hline SCA_4d & 2.84 & 0.00 & 0.19 & 1.00 & 1277.90 & 21.63 & 1046.20 & 16.20 & 1.00 & 1.00 \\
\hline SCA_6d & 2.33 & 0.00 & 0.29 & 1.00 & 1190.50 & 16.75 & 882.70 & 11.33 & 1.00 & 1.00 \\
\hline SCA_8d & 2.87 & 0.00 & 0.16 & 1.00 & 1007.20 & 10.45 & 823.37 & 9.00 & 1.00 & 1.00 \\
\hline SCA_10d & 2.86 & 0.00 & 0.13 & 1.00 & 1047.70 & 59.29 & 742.47 & 20.00 & 1.00 & 1.00 \\
\hline S96_0d & 3.21 & 0.00 & 0.13 & 0.00 & 1058.60 & 0.00 & 853.42 & 11.00 & 1.00 & 1.00 \\
\hline S96_2d & 4.80 & 0.22 & 0.02 & 0.22 & 1324.40 & 141.74 & 1310.10 & 109.23 & 1.00 & 1.00 \\
\hline S96_4d & 2.96 & 0.00 & 0.16 & 0.00 & 1221.10 & 27.79 & 973.61 & 20.50 & 1.00 & 1.00 \\
\hline S96_6d & 3.14 & 0.00 & 0.12 & 0.00 & 800.40 & 3.00 & 800.00 & 3.00 & 1.00 & 1.00 \\
\hline S96_8d & 2.21 & 0.00 & 0.21 & 0.00 & 729.51 & 10.56 & 688.16 & 7.50 & 1.00 & 1.00 \\
\hline S96_10d & 2.27 & 0.00 & 0.19 & 0.00 & 964.38 & 0.00 & 718.91 & 16.00 & 1.00 & 1.00 \\
\hline
\end{tabular}

VS group indicated samples from vineyard soil, GM group indicated samples from group must, S01 group indicated samples from alcohol fermentation inoculated Saccharomyces cerevisiae CEC01, SCA group indicated samples from alcohol fermentation inoculated Saccharomyces cerevisiae CECA and S96 group indicated samples from alcohol fermentation inoculated Saccharomyces cerevisiae 796 

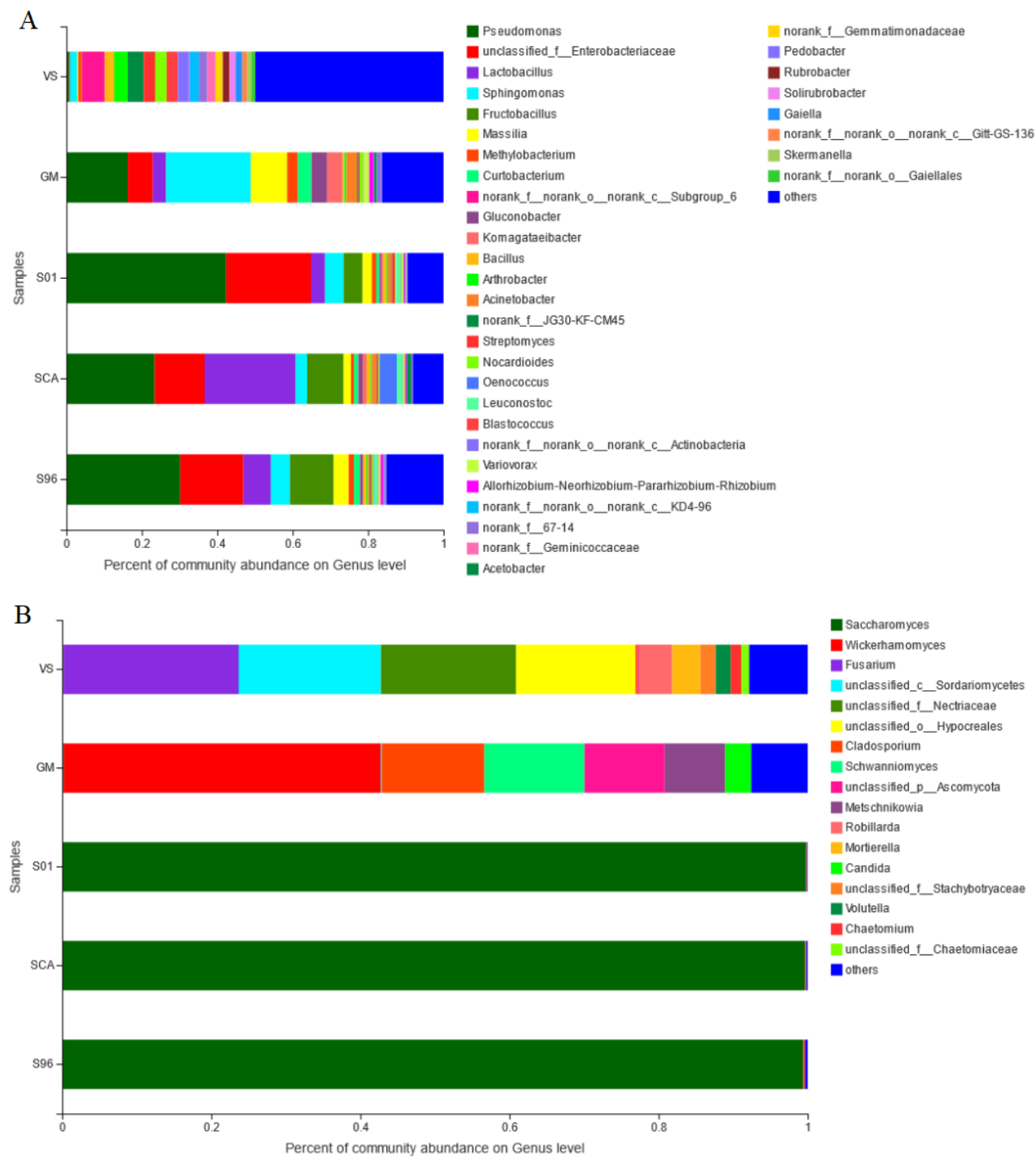

Fig. 1: Average relative abundance of bacterial (A) and fungi (B) at genus level in five sample groups

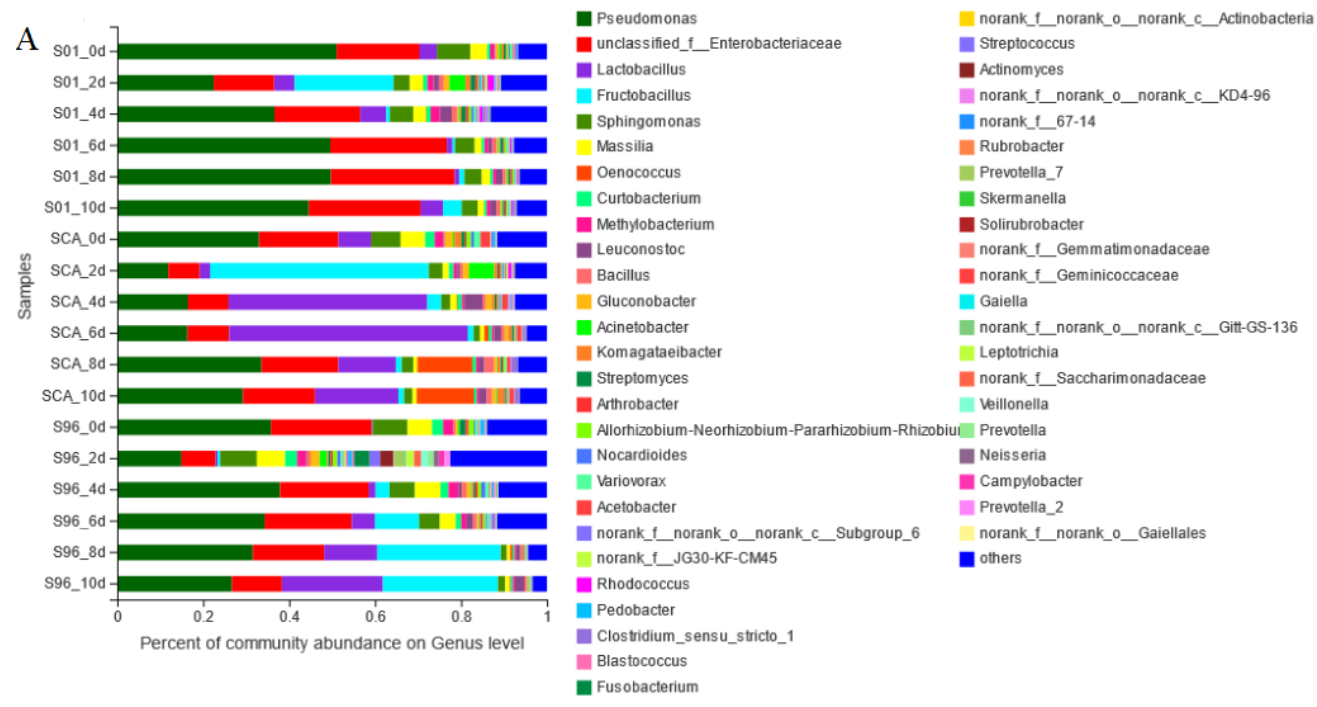




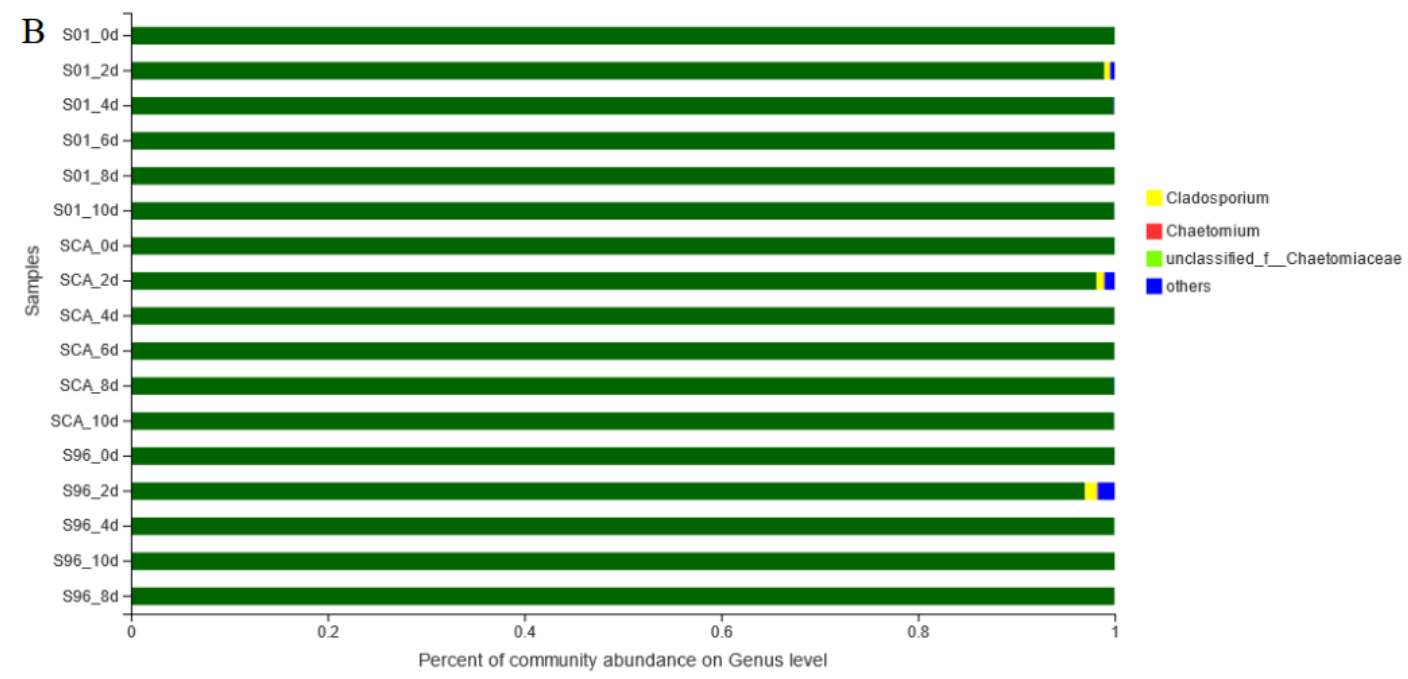

Fig. 2: Characterization of microbial communities on wine samples during fermentation from three fermenters. (A) Relative abundance at the bacterial genus level. (B) Relative abundance at the fungi genus level
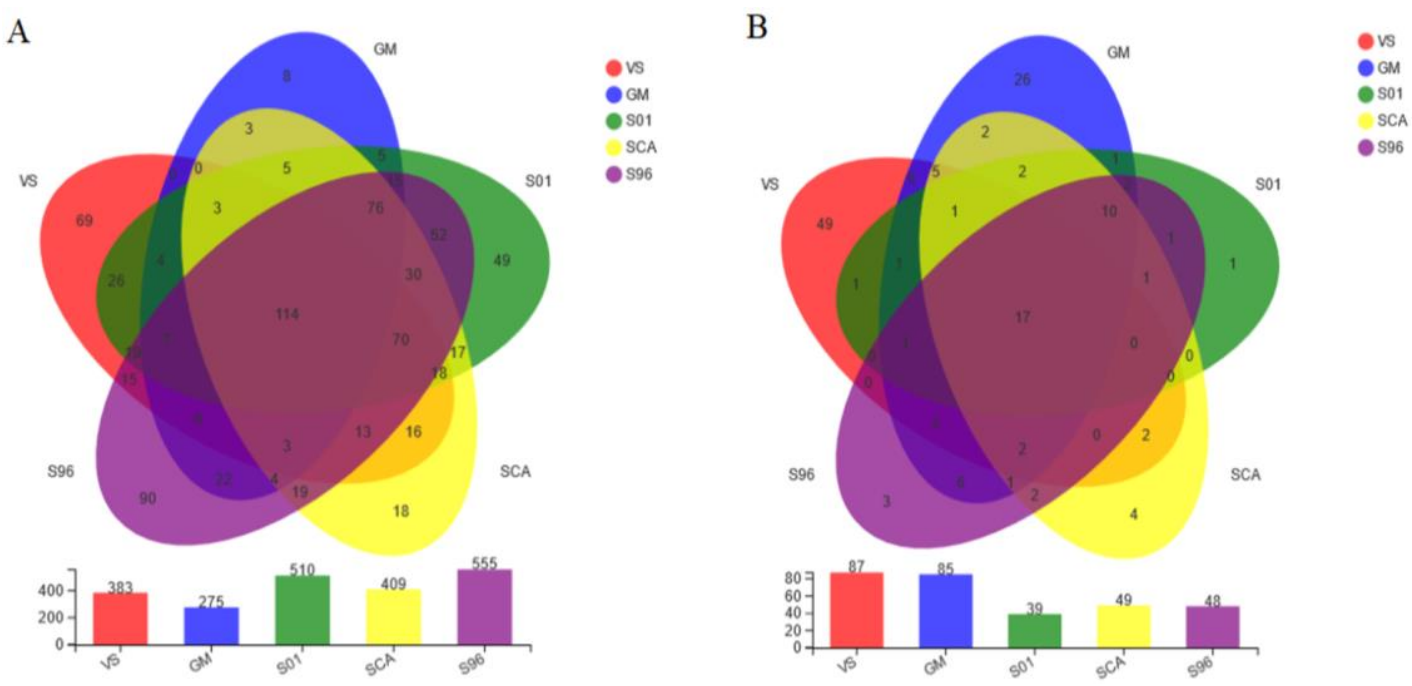

Fig. 3: Venn diagram analyzing unique and shared genera between five sample groups. (A) Bacterial genera. (B) Fungi genera

\section{Beta Diversity and Correlations of Sample Groups}

The beta diversity between fermentation samples were analyzed further using Principal Component Analysis (PCA) and Kruskal-Wallis test was used to detect the genera with differences in the relative abundance of different groups of samples to evaluate the significance of the observed differences.

Evidence of differences in microbial communities between S01, SCA, S96 samples during fermentation were illustrated by the PCA (Fig. 4), in which each dot represented a fermentation sample and samples in the same group were clustered by an ellipse. The shorter for the distance between groups, indicated the higher genera similarity of the microbial communities between the groups. According to the genera-based PCA analysis for bacterial communities (Fig. 4A), 84\% variances were illustrated by the two axes and the ellipses of S01, SCA, S96 group samples on the PCA plot were overlapped, indicating that their bacterial communities were similar. In the same group, samples from different fermentation time periods were scattered in the ellipse, which suggested that the bacterial structure was highly affected by fermentation time. The genera-based PCA for fungal communities (Fig. 4B) demonstrated that fungal communities of S01, SCA, S96 group were largely similar. Especially the ellipses of the S01 and SCA sample groups were almost contained by the ellipses of the S96 sample group, showing that the S96 sample group obtained the most diverse fungi community profile amongst the three sample groups. 
Differences in the relative abundances of the top 25 bacteria genera were also analyzed (Fig. 5). Compared with the S01 group, the abundance of Oenococcus and Tatumella were significantly improved in the SCA group, while the level of Streptomyces was significantly downregulated ( $\mathrm{p}<0.05$, Fig. 5A). Similarly, the level of Oenococcus increased in the SCA group comparison with the S96 group ( $<<0.05$, Fig. 5B). In the S01 group, Komagataeibacter and Streptomyces were found to be of a higher abundance when compared to those of $\$ 96$ group ( $p<0.05$, Fig. 5C). The comparison between grape must and wine fermentation samples revealed abundant bacterial genera with significant differences (Fig. 5D, 5E, $5 F)$. For example, at the significant level of 0.001 , a trend was observed on the S01 group, with decreased Methylobacterium, Sphingomonas, Pedobacte and Massilia comparison with the GM group, meanwhile norank $f \_$Mitochondria, Curtobacterium, Variovorax, Frigoribacterium and Nocardioides were identified as being differentially abundant in the GM group comparison with the S01 group $(\mathrm{p}<0.01)$. The Sphingomonas, Massilia, Curtobacterium and Frigoribacterium of the GM group were higher than those of the SCA group $(\mathrm{p}<0.01)$. For S96 sample group, the bacterial genera that were significantly different from the grape must group were Sphingomonas, Variovorax and Curtobacterium. Generally, there were more significant differences in some bacterial genera between crushed grape must and fermentation samples inoculated with $S$. cerevisiae.

The difference in fungi genera between different groups was really not complicated compared with

A

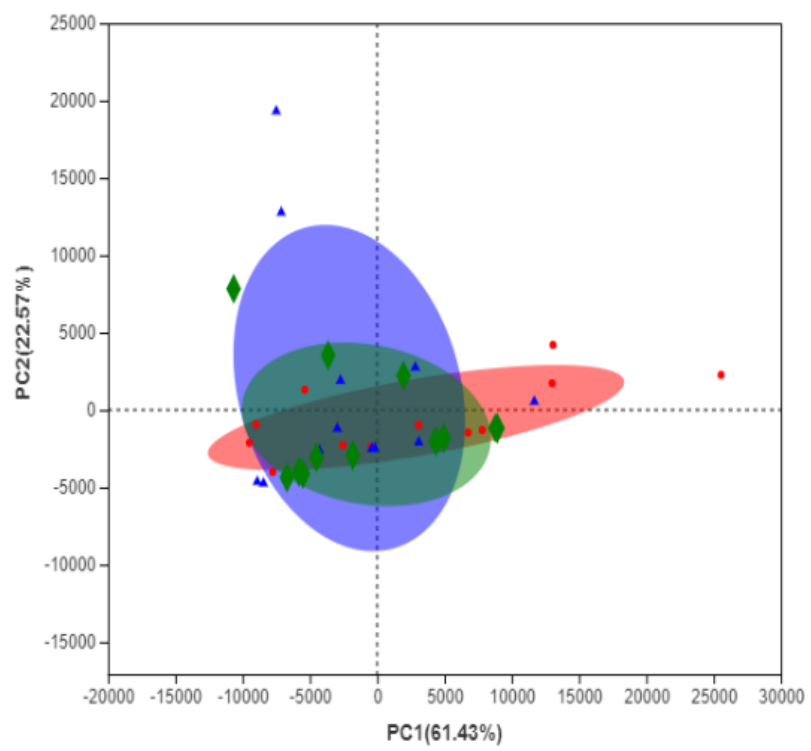

bacterial genera (Fig. 6). There was no significant difference in the level of top 25 fungi genera among the three fermentation samples inoculated with different $S$. cerevisiae and Saccharomyces was the most abundant genera (approximately 99\%) in S01, SCA and S96 group. However, the levels of all identified fungi genera were significantly higher in the GM group than those of S01, SCA and S96 samples, except for Saccharomyces. Saccharomyces and Wickerhamomyces, which were two dominant taxa in the 25 most abundant genera at each fermentation process.

\section{Analysis of Key Microbial Communities}

The biomarkers of bacteria genera in S01, SCA and S96 groups were determined using LEfSe analysis. A cladogram demonstrated that the significant changes in taxa $(p \leq 0.05$, LDA score $>2)$ during the linear discriminant analysis (LDA) test (Fig. 7). These differential genera including Komagataeibacter, Micromonospora, Streptomyces, Brevibacterium and Agromyces were prevalent in the S01 group versus the SCA and S96 groups. Fermentation inoculated with $S$. cerevisiae CECA increased the relative abundance of Lactobacillus and Oenococcus, other enriched bacterial genera also belonged to the family Bacilli. There were no significant differences in bacterial genera in the S96 group and family Ruminococcaceae were dominant in the S96 group. The biomarkers of fungi genera in S01, SCA and S96 groups were not detected using LEfSe analysis.

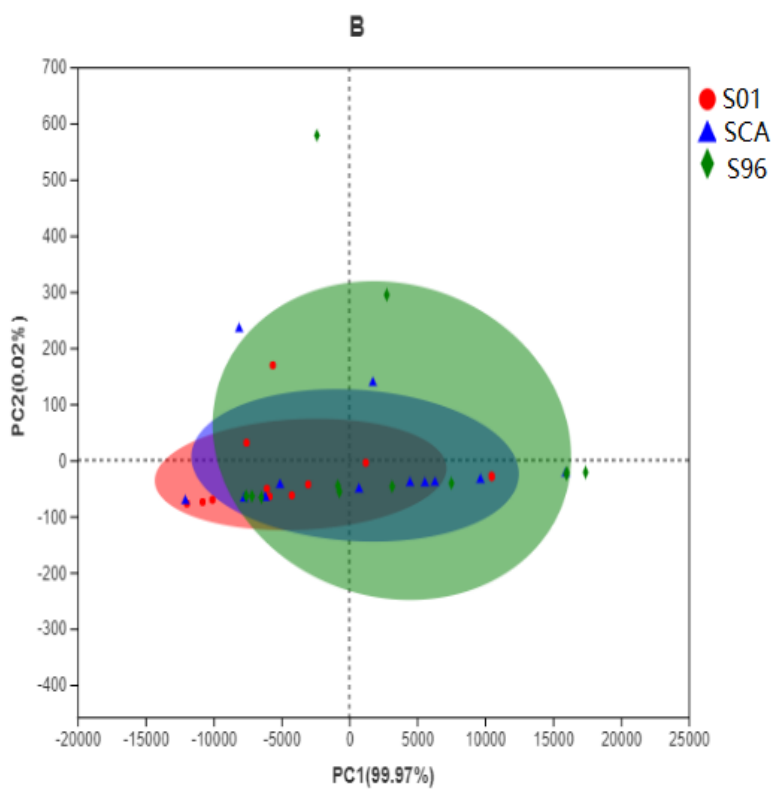

Fig. 4: Principle Component Analysis (PCA) of microbial communities on wine fermentation samples inoculated with three different Saccharomyces cerevisiae. (A) Bacterial genera. (B) Fungi genera 


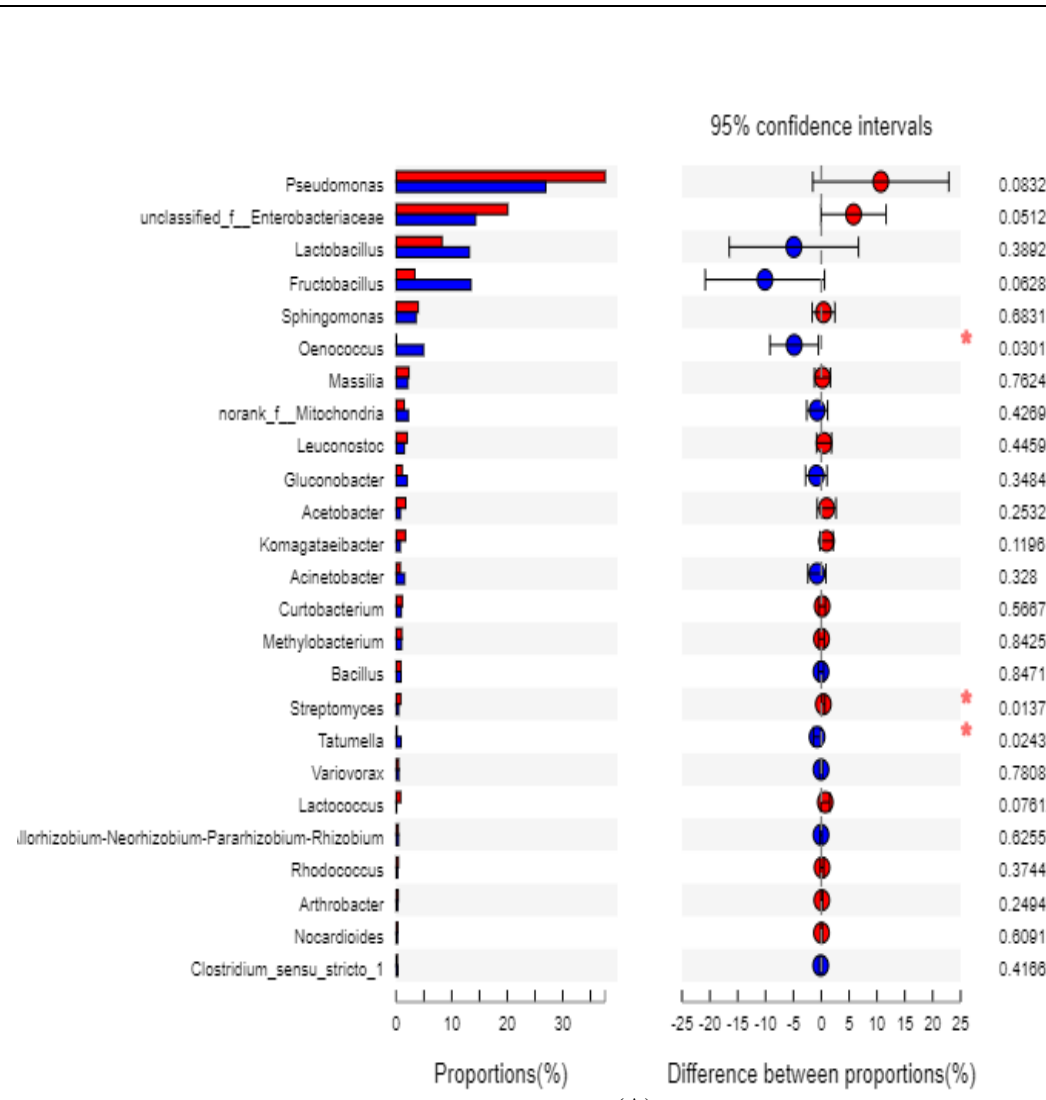

(A)

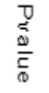

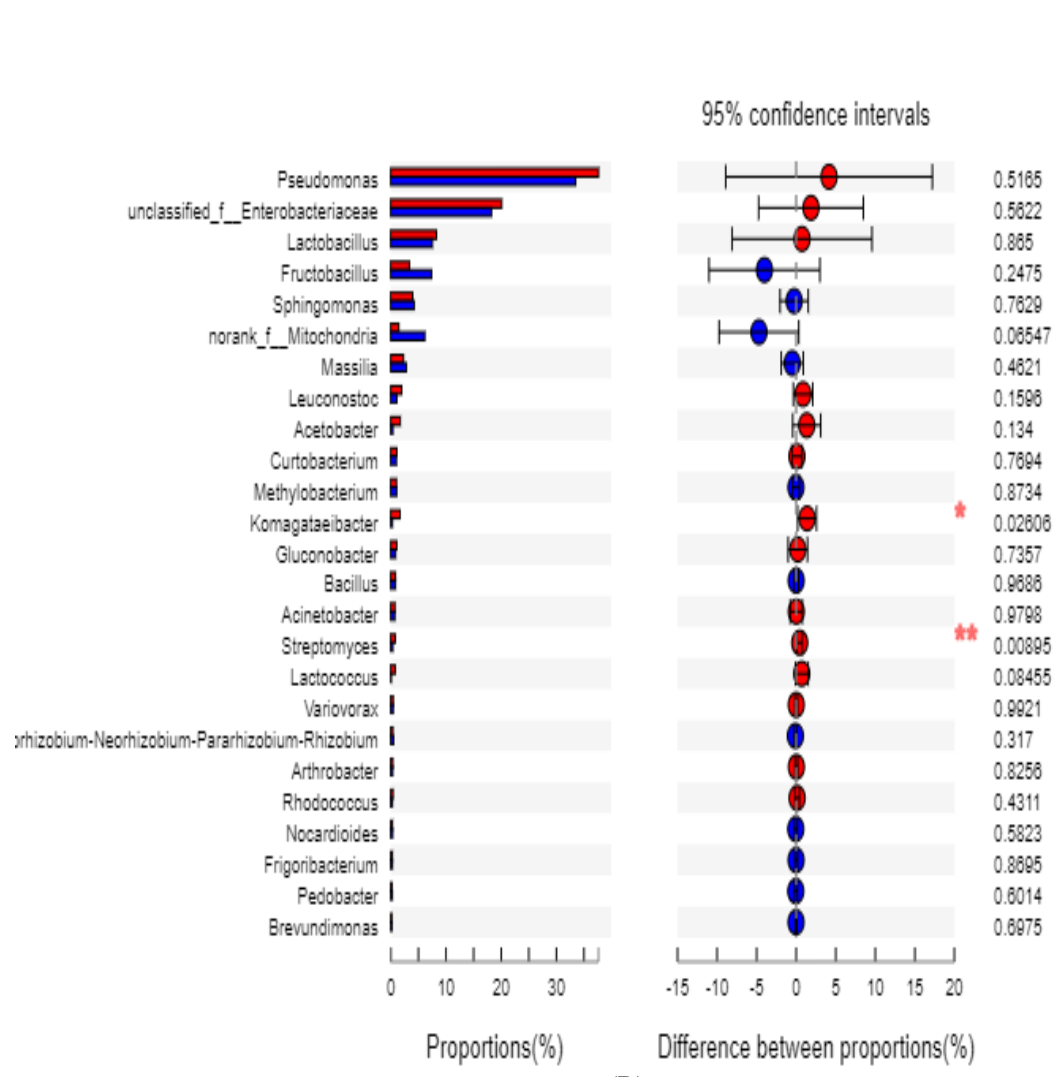

$55 \%$ confidence intervals

(B) 

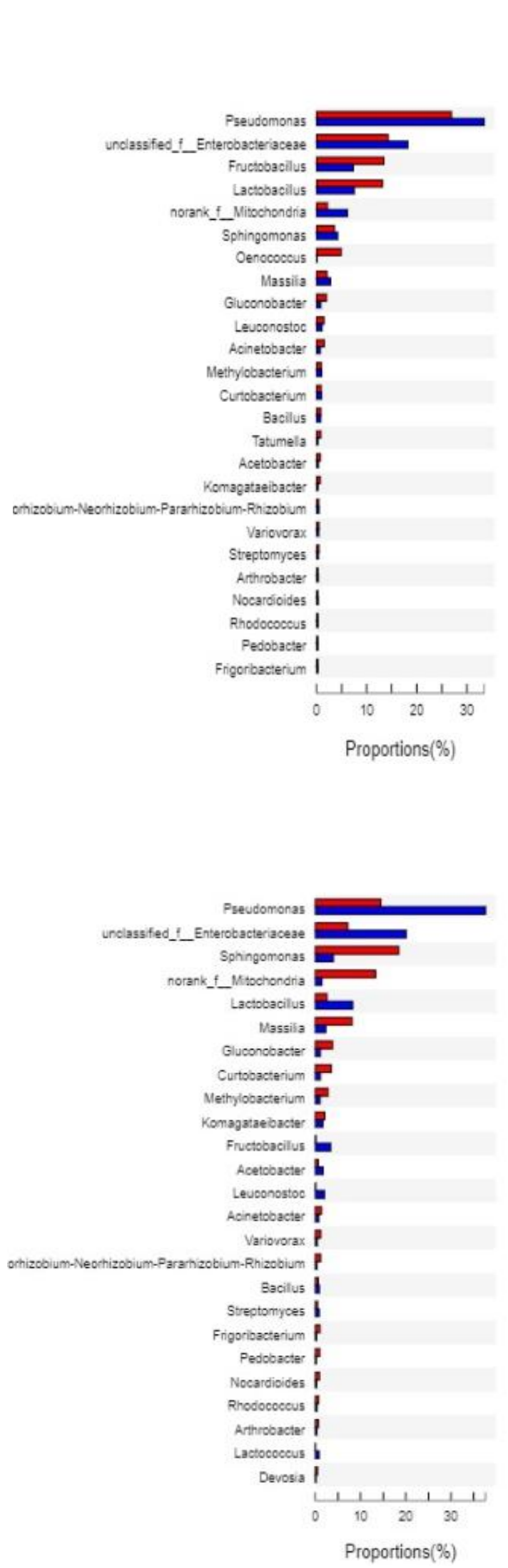

(C)

$95 \%$ confidence intervals

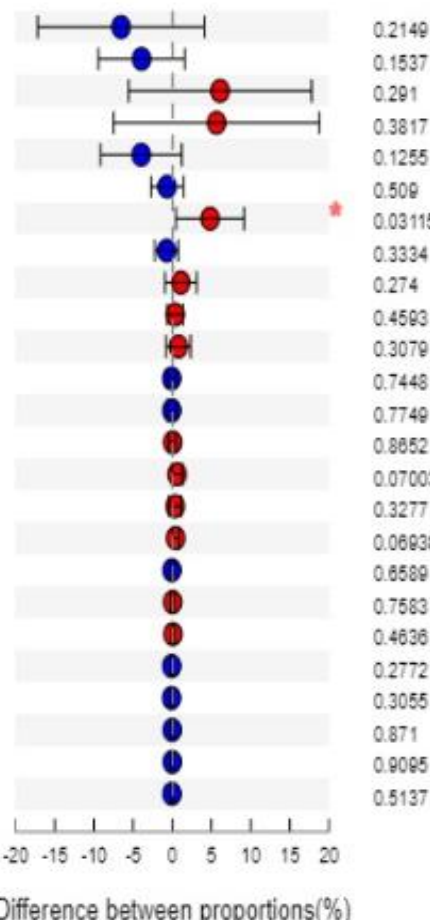



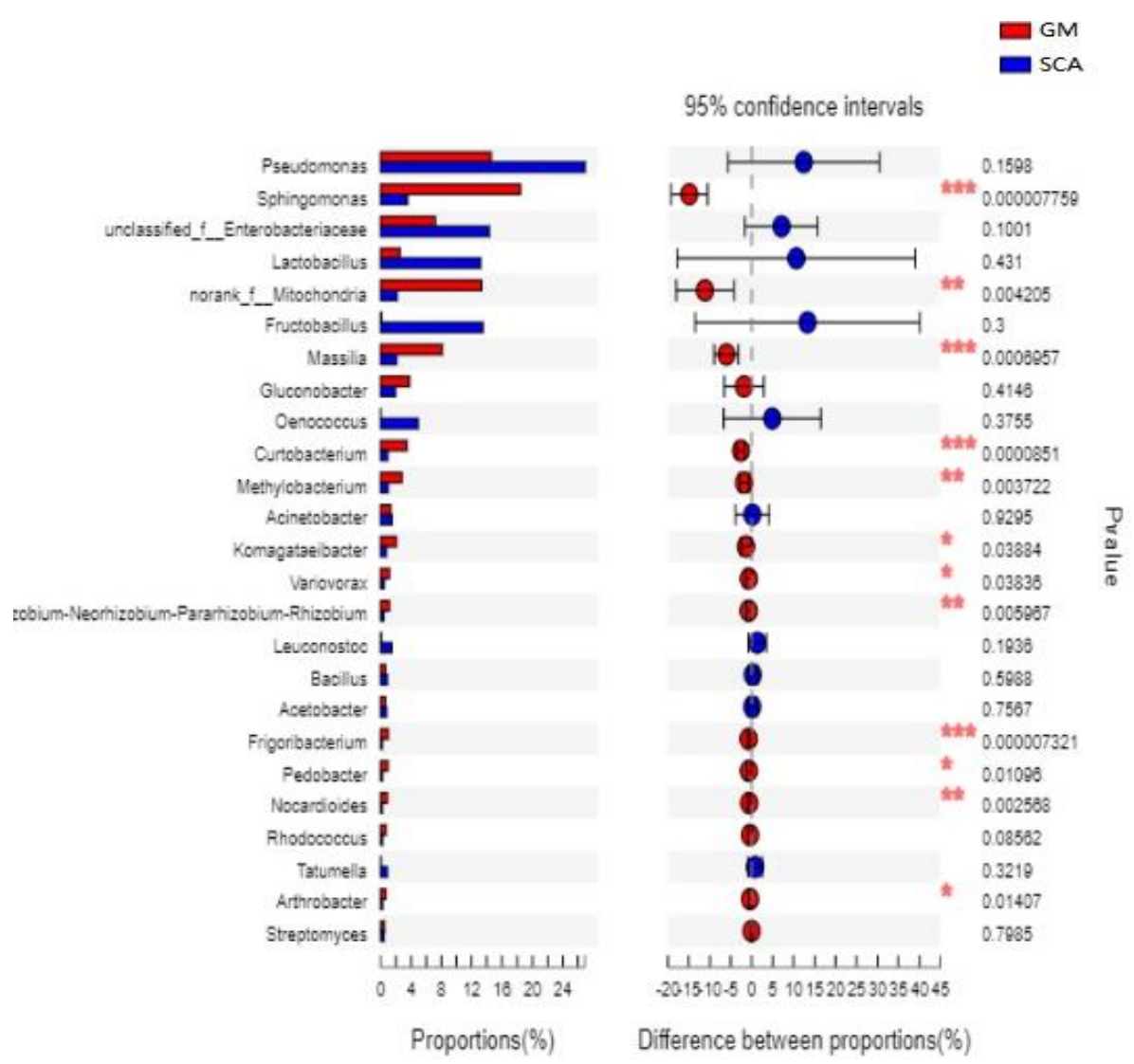

(E)
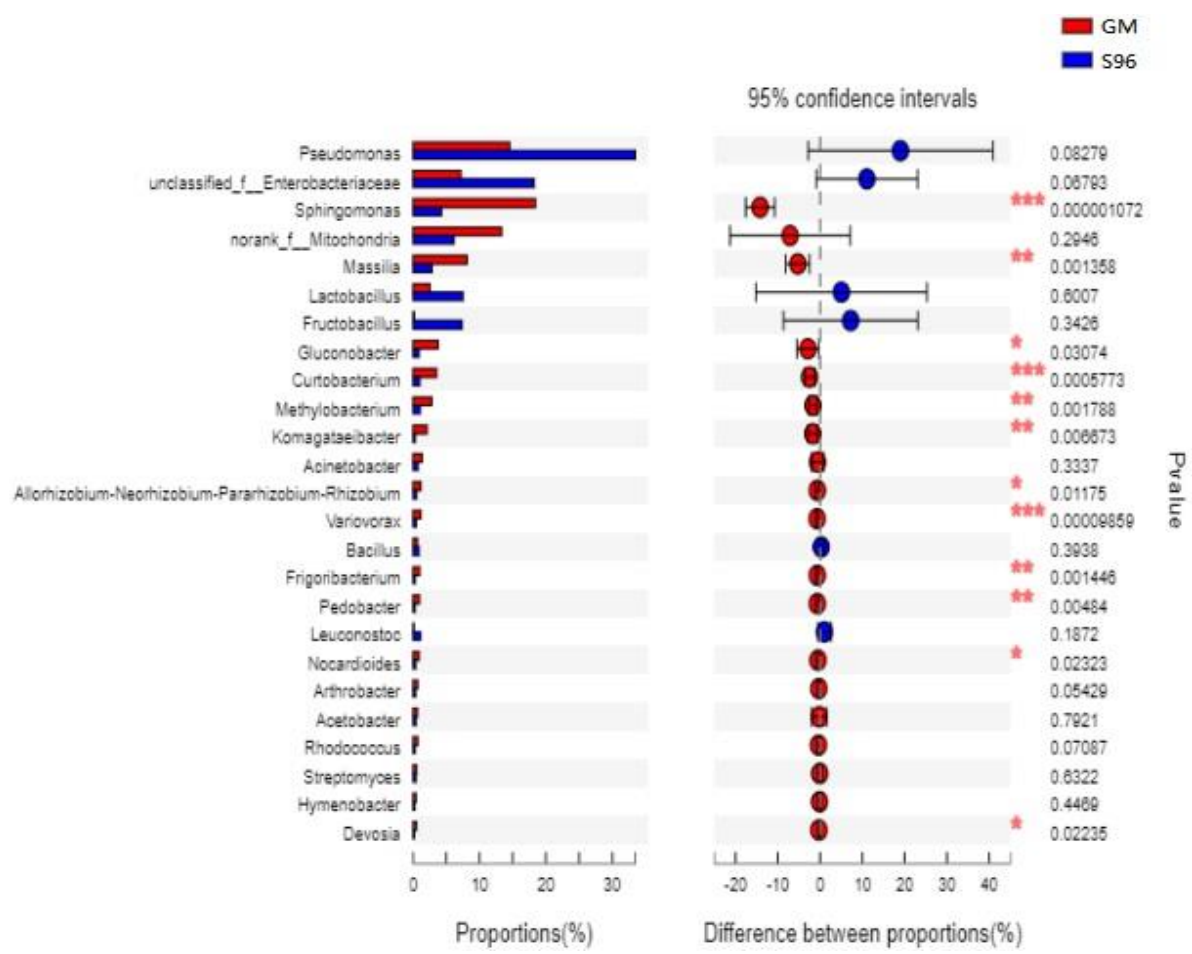

(F)

Fig. 5: Comparison of the top 25 bacteria genera between grapes must and wine fermentation samples groups. The stars indicated significance levels: ‘***’0.001'**’ 0.01, '*’0.05 

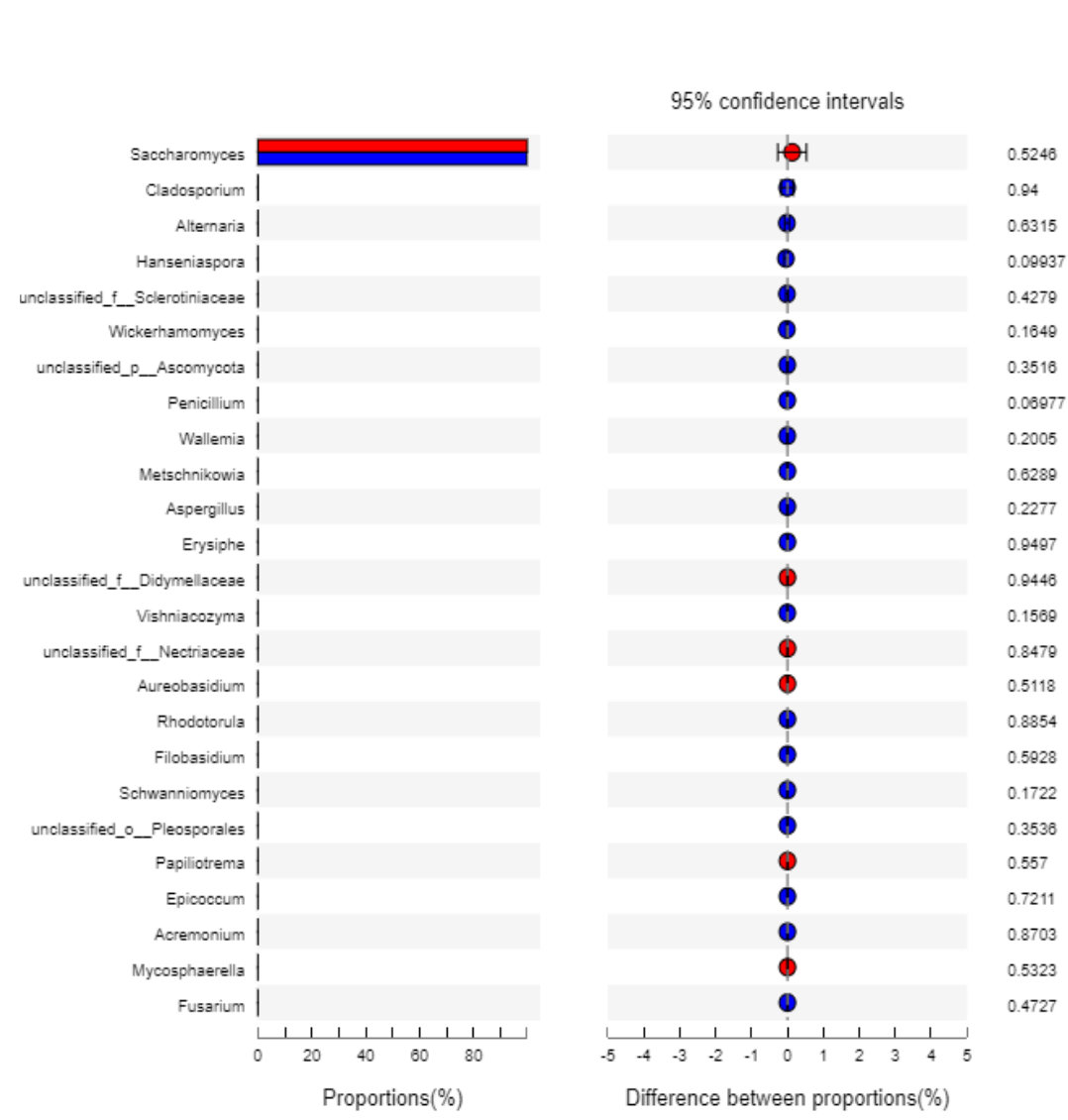

rence between proportions $(\%)$

(A)

$95 \%$ confidence intervals
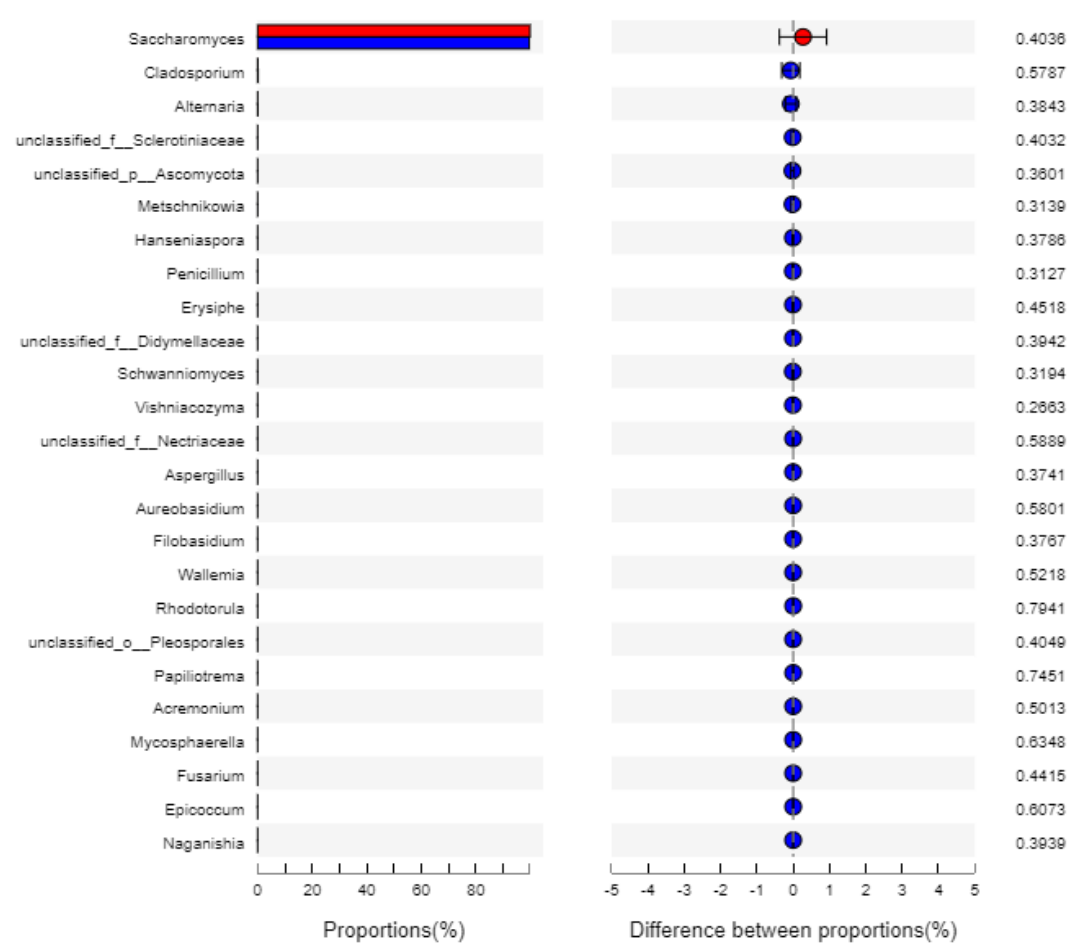


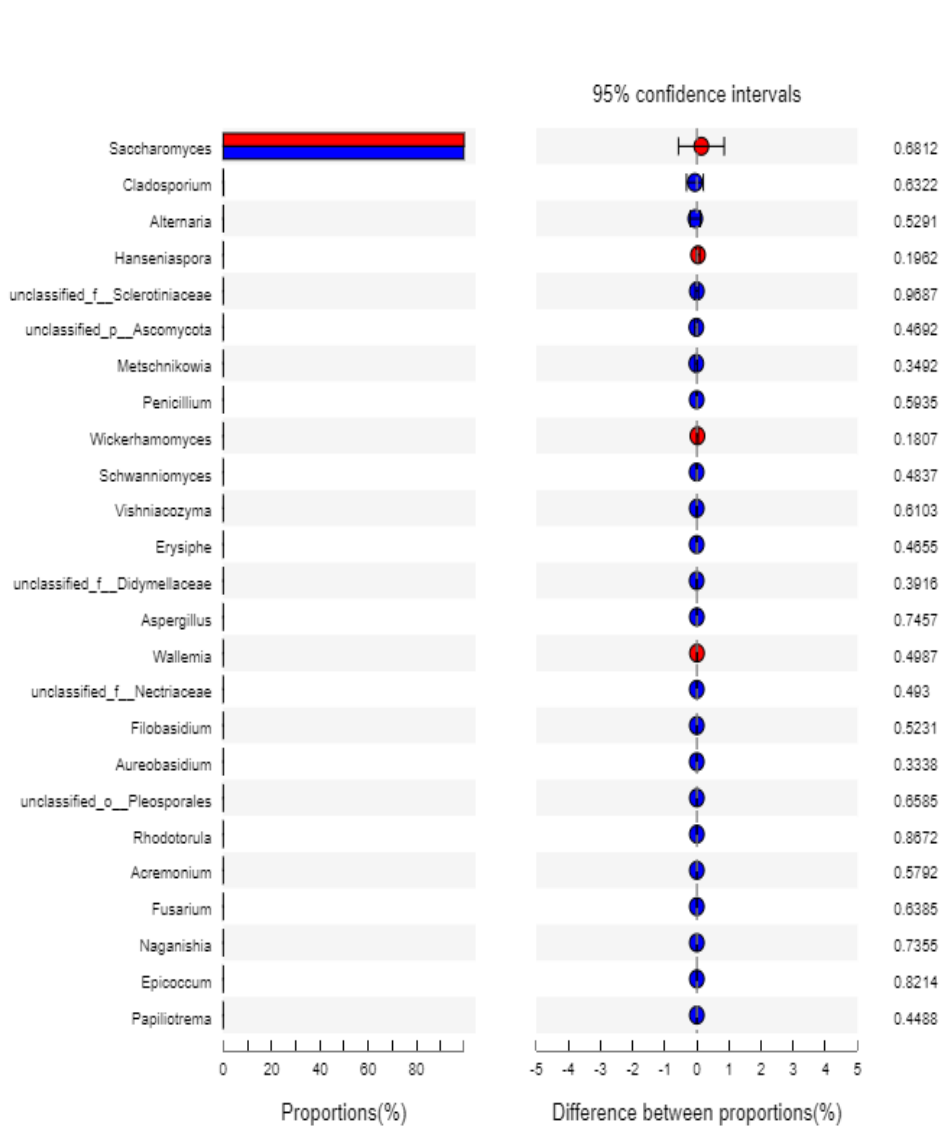

(C)

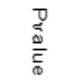
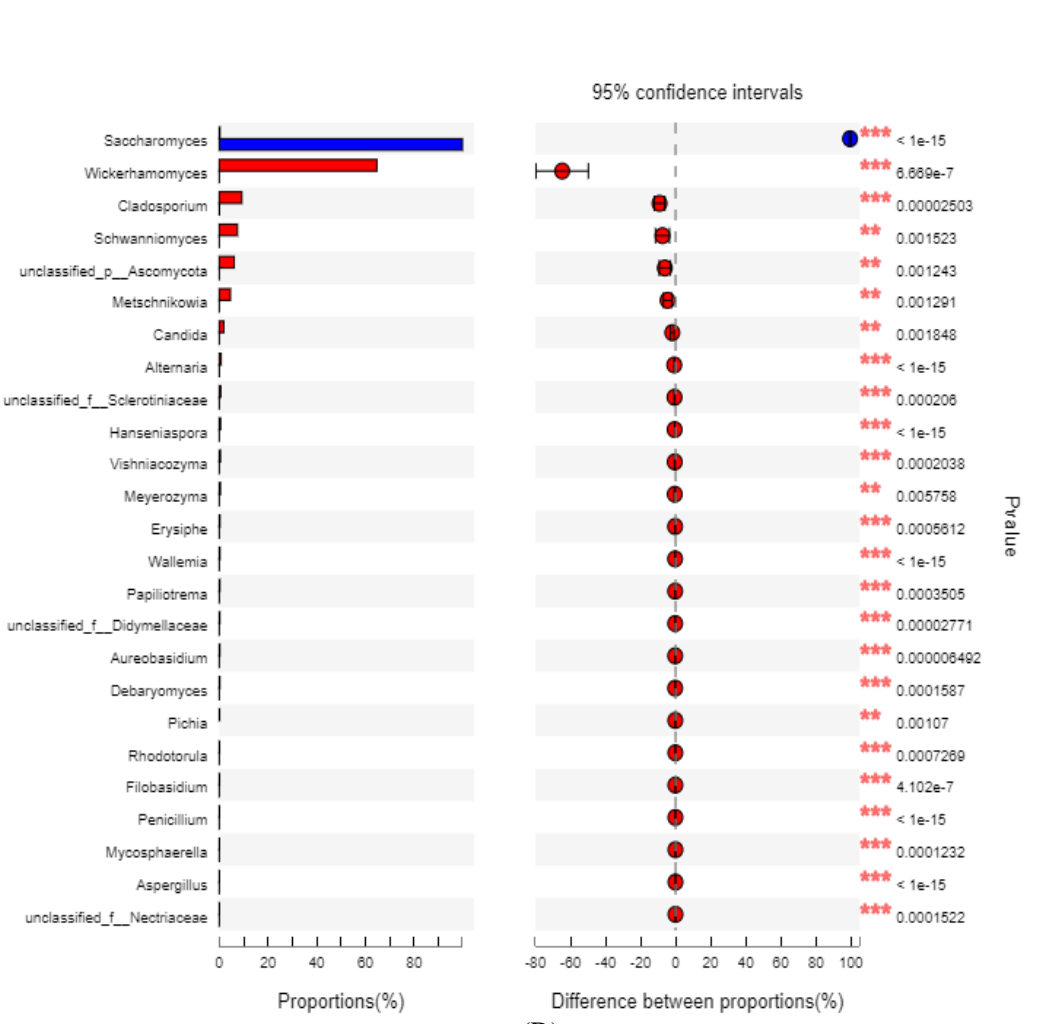

(D) 

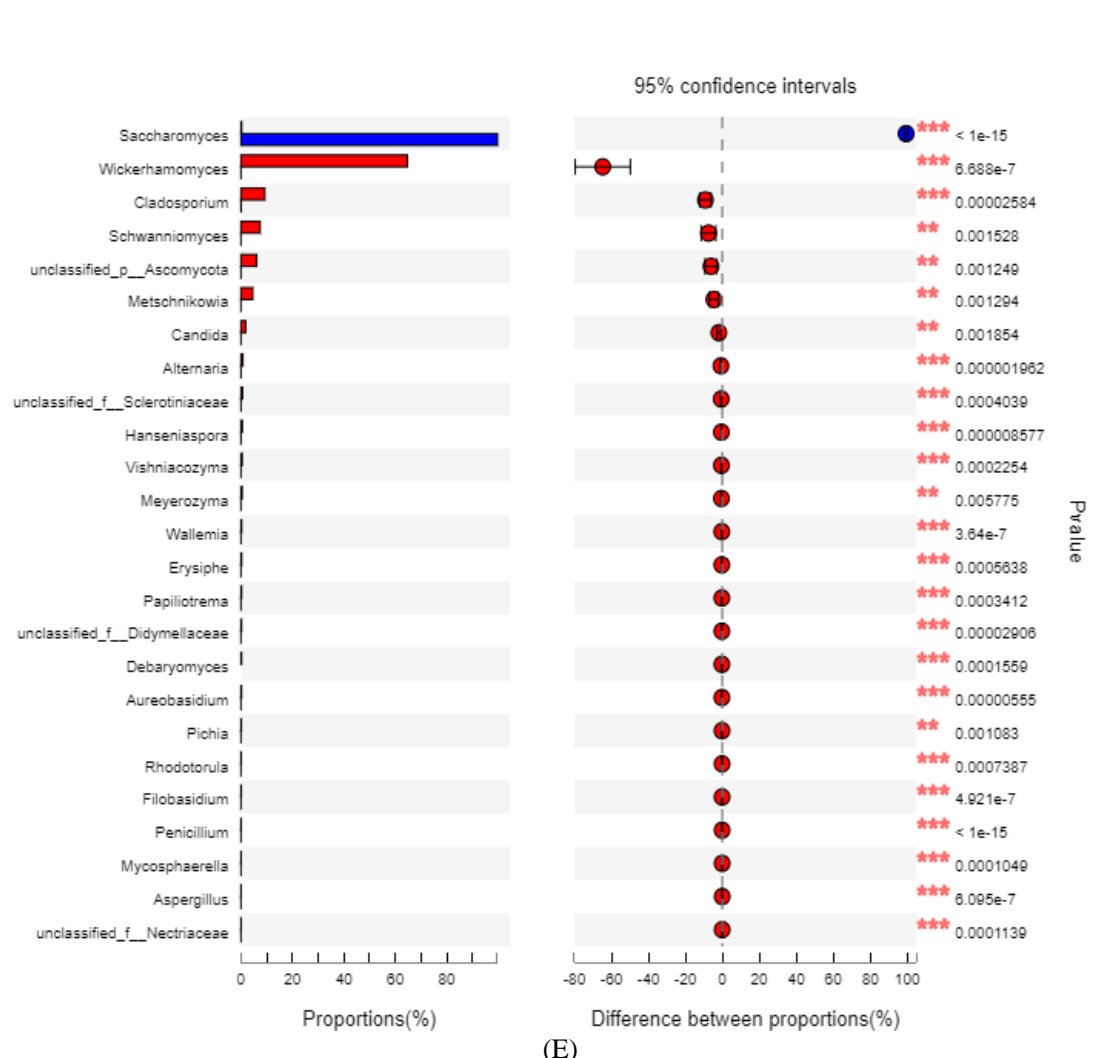

घ

CFB

(E)
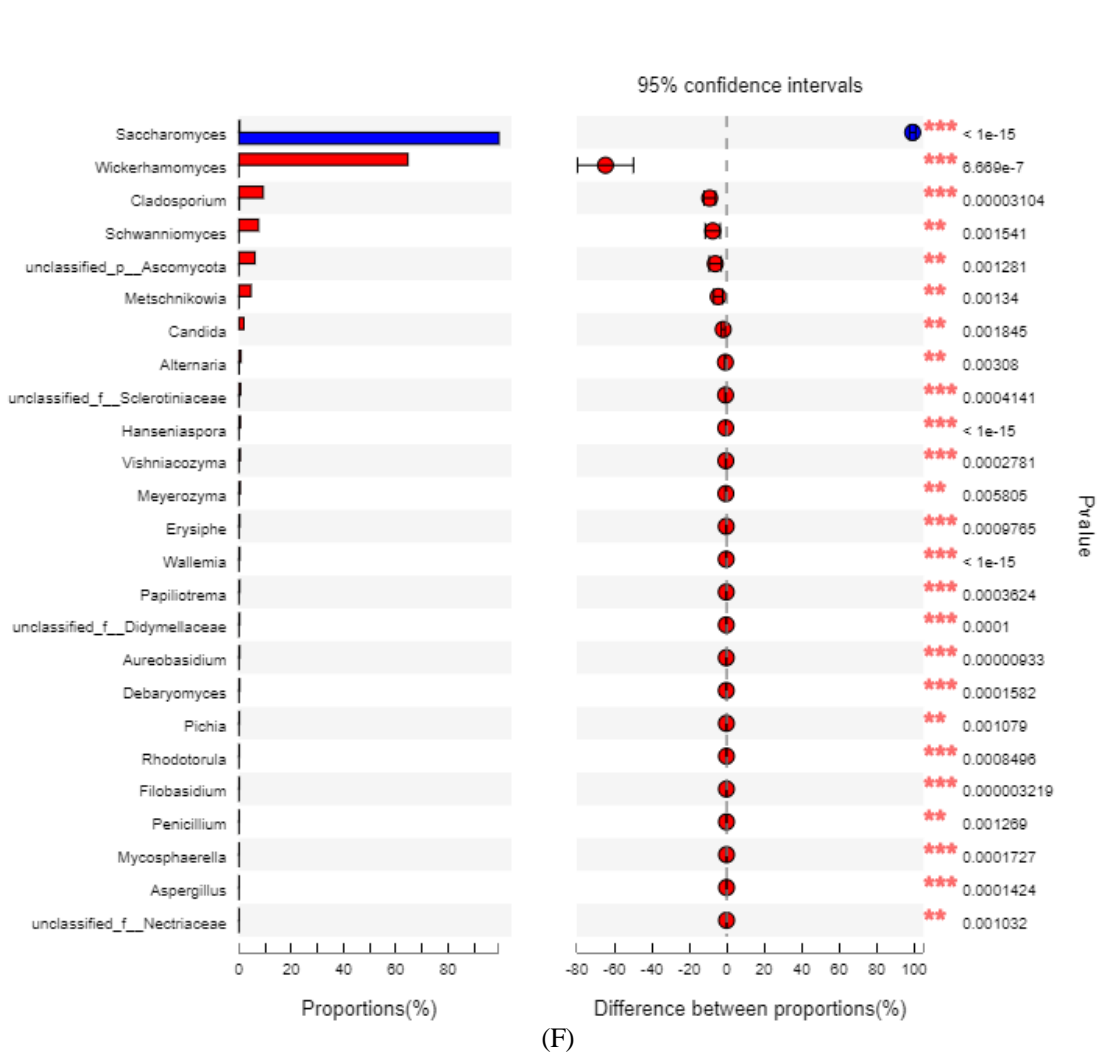

Fig. 6: Comparison of the top 25 fungi genera between grapes must and wine fermentation samples groups. The stars indicated significance levels: ‘***’0.001'**’0.01, ‘*’0.05 

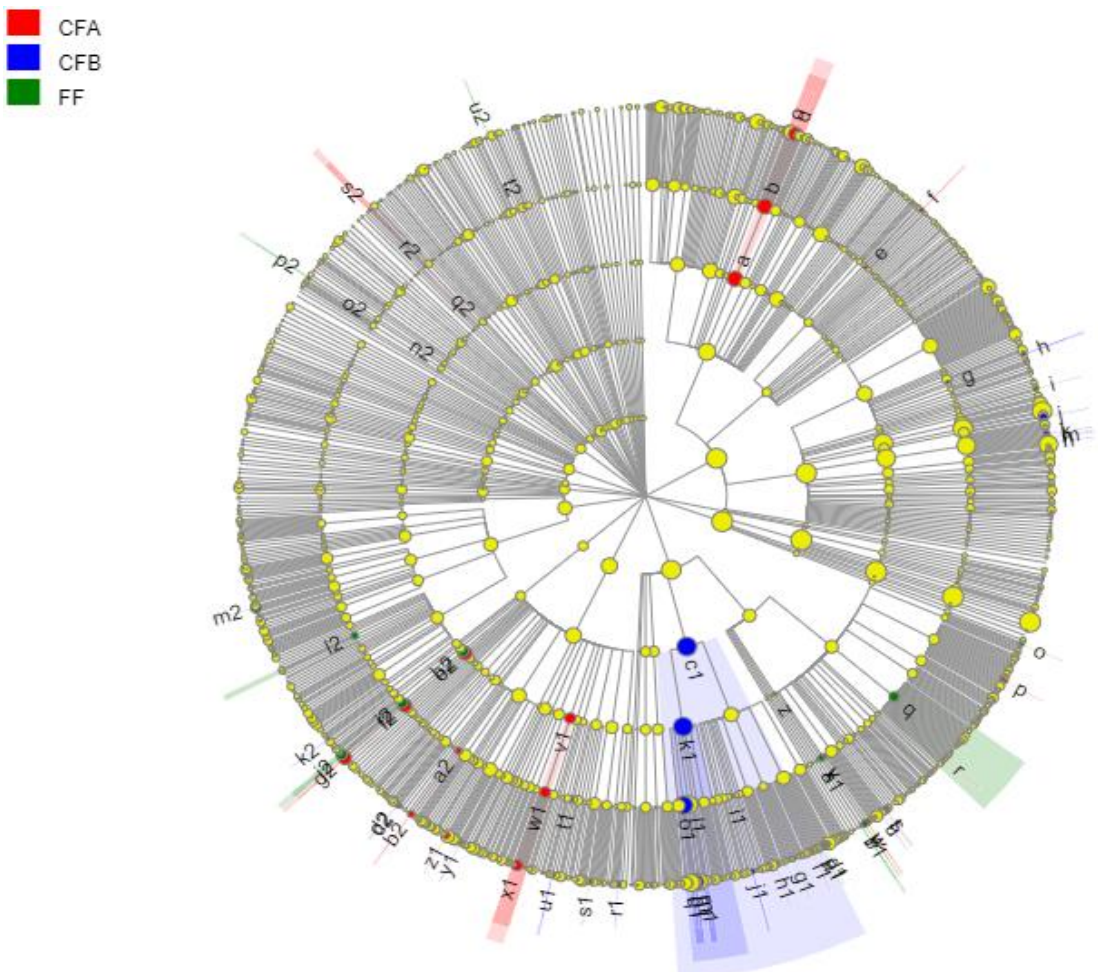

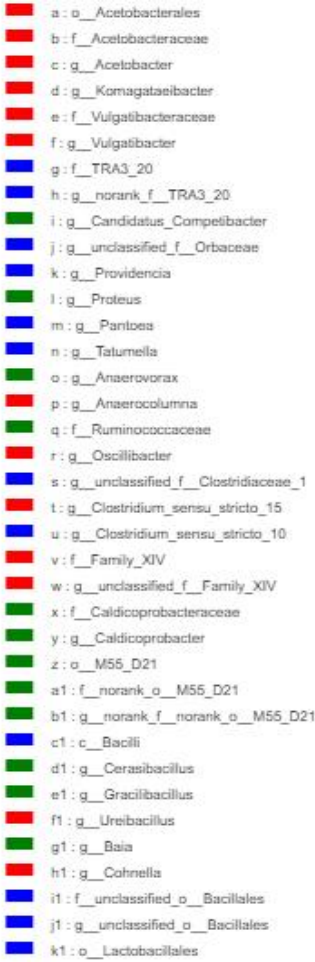

Fig. 7: Analyses of the significant changes in taxa at different taxonomic levels in S01, SCA, and S96 groups during fermentation. Cladogram of bacterial LEfSe analysis $(\mathrm{p} \leq 0.05$, LDA score $>2)$

\section{Discussion}

In the current wine industry, the growing demand from consumers has apeared for the "terroir" with distinct flavour characteristics in which the microflora structures have a fundamental effect. Capozzi et al. (2015) have reviewed the microbial terroir and food innovation and pointed that the natural biodiversity of microbial communities as well as correlated to each specific terroir showed a unique composition and represent a great resources for the winemaking improving. Therefore, in the present study, the microbial communities diversity of vineyard soil, grape must and wine fermentation inoculated with different strains of $S$. cerevisiae were comprehensively investigated based on the results of high-throughput sequencing technology.

Current studies have shown that the microbial community diversity of wine grape would be influenced by many elements such as geography, climate, grape variety and viticultural practice (Martins et al., 2013; Gao et al., 2019). Whereas, it is well accepted that vineyard soil would exert a major and an independent impact on the quality and varietal typicity of grape and wine, therefore the wine-makers would prefer to choose vineyards with good environmental conditions (geography and climate) to produce high-quality wine (Van Leeuwen and Seguin, 2006; Gómez-Míguez et al.,
2007). Soil texture, organic matter, mineral composition and other physical and chemical properties could influence the growth and distribution of microorganisms, which would also in turn change the composition of the microbial community in grape and wine products (Girvan et al., 2003). Shacheng (Hebei, China) is one of the oldest grape-cultivation regions for Cabernet Sauvignon grapes in China. It is located in $40^{\circ}$ north latitude with sandy soil, a great temperature difference between day and night for nearly $11-12.4^{\circ}$, a high percentage of sunshine $(68 \%)$ and a hot rainy senson, which made it into a golden zone of grape-growing in the world. Previous studies have been investigated the microbial community, volatile compounds and charactiristics of grape and wine from this area, while there is few information about the relationship of microbial community between the soil, grape and wine (Lu et al., 2020; Zhang et al., 2017). In the present study, the bacterial and fungi compositions of Shacheng vineyard soil were analyzed. The predominate bacateria and fungi were norank_f_JG30-KF-CM45, Arthrobacter, Streptomyces, Actinobacteria and Fusarium, unclassified-cSordariomycetes, unclassified-f-Nectriaceae, unclassified-oHypocreales. While, these results are varies compared with the microbial community of vineyard soil from the reported literature. For example, del Carmen Portillo et al. (2016) reported that Oenococcus, Streptococcus and Acinetobacter were the dominant genera in East-oriented 
vineyard must, Bacillus dominated in South-oriented vineyards must, Erwinia and Acinetobacter were enriched in flat or not-oriented vineyards. Wei et al. (2018) showed that Ascomycota, Tetracladium, Sordariales and Geomyces were the predominant fungi genera in soil samples obtained from three winery regions in Xinjiang Province (China). Rivas et al. (2022) demonstrated that a predominance of the phyla Proteobacteria and Actinobacteria for the bacteria in the soil and rhizosphere samples and Ascomycota and Basidiomycota were the most abundant phyla for the fungal communities in considered re-emerging grapevines in Argentine. From these findings, the soil microbial communities from different regions had a large differences, which mainly related to the geography, local climatic conditions and viticultural practice as the previous study reported.

Compared to the vineyard soil, the bacterial community structures in GM were less consistent and complex. Among them, Pseudomonas, Sphingomonas, norank_f_Mitochondria, Massilia and unclassified_f_Enterobacteriaceae occupied about $50 \%$ of the total bacterial genera Fig. 1A. For fungus distribution, Fusarium, unclassified_c_Sordariomycetes, unclassified_f_Nectriaceae and unclassified_o_Hypocreales in soil had higher abundances Fig. 1B.

Besides, we also exaimed the relationships between the grape must and fermented wine samples and found that Pseudomonas, unclassified_f_Enterobacteriaceae, Sphingomonas, Lactobacillus and Oenococcus were shared by the four sets of sample. These results were consistent with previous reports that Oenococcus, the lactic acid bacteria, was the dominant and indispensable genus during winemaking with the function of malic acid fermentation conferring unique sensory properties (Ferrando et al., 2020). Perazzolli et al. (2014) observed Pseudomonas, Erwinia and Acetobacter in grapevine plants and inferred that these genera would be easily transferred to grape berries, in which Pseudomonas could act as biological disease inhibitors to promote plant growth and health. The starter of $S$. cerevisiae is inoculated in the grape must to form a high population and complete a well-controlled fermentation. Under some circumstances, if the $S$. cerevisiae strain can't successfully compete with the native strains, which would cause the fermentation to be unable to proceed in the expected direction (Fleet, 2008; Santamaría et al., 2005). In this study, we observed that $S$. cerevisiae dominated all the fermentation process after inoculation (Fig. 3B). For the bacterial community, the distribution of the main genera under the three fermentation methods was similar, including Pseudomonas, unclassified_f_Enterobacteriaceae and Lactobacillus. Chen et al. (2020) pointed that the relative abundances of Pseudomonas and Massilia gradually decreased during ice wine fermentation process made from Vidal grapes. The differences between three fermentation processes were realized as the inconsistent change trend of certain microorganisms or the different key microorganisms. For example, Komagataeibacter, Micromonospora, Streptomyces, Brevibacterium and Agromyces were prevalent in S01 group versus the SCA and S96 groups; fermentation inoculated with $S$. cerevisiae CECA increased the relative abundance of Lactobacillus and Oenococcus; and there were no significant differences in bacterial genera in S96 group, in which family Ruminococcaceae were dominan.

\section{Conclusion}

In this study, the high-throughput sequencing technique was performed to analyze the microbial community diversity of soil, grape juice and wine production. We found that the bacterial composition of soil was diverse with other genera (relative abundance $<1 \%$ ) accounting for $51.06 \%$ and a higher abundances for fungus distribution. Across all must and wine samples, the bacterial genera detected were largely members of the Pseudomonas, unclassified_f_Enterobacteriace, Lactobacillus, Fructobacillus and Sphingomona. Principle component analysis showed the microbiota structures between S01, SCA and S96 fermentation were similar and the major bacterial genera were Pseudomonas, unclassified_f_Enterobacteriaceae and Lactobacillus, whereas the major fungi genera were Saccharomyces. The core microorganisms in the S01 group were Komagataeibacter, Micromonospora, Streptomyces, Brevibacterium and Agromyces, SCA fermentation increased the relative abundance of Lactobacillus and Oenococcus, family Ruminococcaceae was dominant in the S96 group. The distinctions in fungi communities between S01, SCA and S96 group were not observed during the fermentation. In the future work, it may be interesting to link the observed microbial community changes with the differences in the macromolecular substances of wine and to use this knowledge to improve the sensory and chemical sensory characteristic of wine in Shacheng.

\section{Acknowledgement}

This work was supported by the Key Research and Development Program of Hebei Province [grant number 19227120D].

\section{Author's Contributions}

Rongbin Li: Has contributed in the experiment, paper writing and publication.

Xu Shi, Haoran Wang, Xiaofang Fu, Huanxiang

Wang: Have assisted in the experiment.

Yaqiong Liu, Jianlou Mu and Jie Wang: Have reviewed and revised the manuscript. 


\section{Ethics}

This article is original and contains unpublished material. The corresponding author confirms that all of the other authors have read and approved the manuscript and no ethical issues involved

\section{References}

Aponte, M., \& Blaiotta, G. (2016). Selection of an autochthonous Saccharomyces cerevisiae strain for the vinification of "Moscato di Saracena", a southern Italy (Calabria Region) passito wine. Food Microbiology, 54, 30-39. doi.org/10.1016/j.fm.2015.10.019

Barata, A., Malfeito-Ferreira, M., \& Loureiro, V. (2012). The microbial ecology of wine grape berries. International Journal of Food Microbiology, 153(3), 243-259. doi.org/10.1016/j.ijfoodmicro.2011.11.025

Capozzi, V., Garofalo, C., Chiriatti, M. A., Grieco, F., \& Spano, G. (2015). Microbial terroir and food innovation: The case of yeast biodiversity in wine. Microbiological Research, 181, 75-83. doi.org/10.1016/j.micres.2015.10.005

Chen, Y., Zhang, W., Yi, H., Wang, B., Xiao, J., Zhou, X., .. \& Shi, X. (2020). Microbial community composition and its role in volatile compound formation during the spontaneous fermentation of ice wine made from Vidal grapes. Process Biochemistry, 92, 365-377. doi.org/10.1016/j.procbio.2020.01.027

de Castilhos, M. B. M., Betiol, L. F. L., de Carvalho, G. R., \& Telis-Romero, J. (2018). Experimental study of physical and rheological properties of grape juice using different temperatures and concentrations. Part II: Merlot. Food Research International, 105, 905-912. doi.org/10.1016/j.foodres.2017.07.075

Ferrando, N., Araque, I., Ortís, A., Thornes, G., BautistaGallego, J., Bordons, A., \& Reguant, C. (2020). Evaluating the effect of using non-Saccharomyces on Oenococcus oeni and wine malolactic fermentation. Food Research International, 138, 109779. doi.org/10.1016/j.foodres.2020.109779

Fleet, G. H. (2008). Wine yeasts for the future. FEMS Yeast Reserach, 8, 979-995. doi.org/10.1111/j.1567-1364.2008.00427.x

Gao, F., Chen, J., Xiao, J., Cheng, W., Zheng, X., Wang, B., \& Shi, X. (2019). Microbial community composition on grape surface controlled by geographical factors of different wine regions in Xinjiang, China. Food Research International, 122, 348-360. doi.org/10.1016/j.foodres.2019.04.029

Girvan, M. S., Bullimore, J., Pretty, J. N., Osborn, A. M., \& Ball, A. S. (2003). Soil type is the primary determinant of the composition of the total and active bacterial communities in arable soils. Applied and Environmental Microbiology, 69(3), 1800-1809. doi.org/10.1128/AEM.69.3.1800-1809.2003
Gómez-Míguez, M. J., Gómez-Míguez, M., Vicario, I. M., \& Heredia, F. J. (2007). Assessment of colour and aroma in white wines vinifications: Effects of grape maturity and soil type. Journal of Food Engineering, 79(3), 758-764. doi.org/10.1016/j.jfoodeng.2006.02.038

Jiang, B., Xi, Z., Luo, M., \& Zhang, Z. (2013). Comparison on aroma compounds in Cabernet Sauvignon and Merlot wines from four wine grapegrowing regions in China. Food Research International, 51(2), 482-489. doi.org/10.1016/j.foodres.2013.01.001

Lam, S. S., \& Howell, K. S. (2015). Drosophilaassociated yeast species in vineyard ecosystems. FEMS Microbiology Letters, 362(20), fnv170. doi.org/10.1093/femsle/fnv170

Lewis, G. K., Hardy, A., Wells, M. P., \& Kerslake, F. L. (2021). Using mobile technology to track wine tourists. Annals of Tourism Research Empirical Insights, 2(2), 100022. doi.org/10.1016/j.annale.2021.100022

Liu, D., Xu, J., Cao, Y., Qi, Y., Yang, K., Wei, X., ... \& Fan, M. (2020). Effect of glutathione-enriched inactive dry yeast on color, phenolic compounds and antioxidant activity of kiwi wine. Journal of Food Processing and Preservation, 44(3), e14347. doi.org/10.1111/jfpp.14347

Lu, Y., Sun, F., Wang, W., Liu, Y., Wang, J., Sun, J., ... \& Gao, Z. (2020). Effects of spontaneous fermentation on the microorganisms diversity and volatile compounds during 'Marselan'from grape to wine. LWT, 134, 110193. doi.org/10.1016/j.lwt.2020.110193

Martins, G., Lauga, B., Miot-Sertier, C., Mercier, A., Lonvaud, A., Soulas, M. L., ... \& Masneuf-Pomarède, I. (2013). Characterization of epiphytic bacterial communities from grapes, leaves, bark and soil of grapevine plants grown and their relations. Plos One, 8(8), e73013. doi.org/10.1371/journal.pone.0073013

Morrison-Whittle, P., \& Goddard, M. R. (2018). From vineyard to winery: A source map of microbial diversity driving wine fermentation. Environmental Microbiology, 20(1), 75-84. doi.org/10.1111/14622920.13960

Nikolaou, E., Soufleros, E. H., Bouloumpasi, E., \& Tzanetakis, N. (2006). Selection of indigenous Saccharomyces cerevisiae strains according to their oenological characteristics and vinification results. Food Microbiology, 23(2), 205-211. doi.org/10.1016/j.fm.2005.03.004

Ortiz, M. J., Barrajón, N., Baffi, M. A., Arévalo-Villena, M., \& Briones, A. (2013). Spontaneous must fermentation: Identification and biotechnological properties of wine yeasts. LWT-Food Science and Technology, 50(2), 371-377. doi.org/10.1016/j.lwt.2012.09.019

OIV. (2020). International Organisation of Vine and Wine, https://www.oiv.int/, 2020. 
Perazzolli, M., Antonielli, L., Storari, M., Puopolo, G., Pancher, M., Giovannini, O., ... \& Pertot, I. (2014). Resilience of the natural phyllosphere microbiota of the grapevine to chemical and biological pesticides. Applied and Environmental Microbiology, 80(12), 3585-3596. doi.org/10.1128/AEM.00415-14

del Carmen Portillo, M., Franquès, J., Araque, I., Reguant, C., \& Bordons, A. (2016). Bacterial diversity of Grenache and Carignan grape surface from different vineyards at Priorat wine region (Catalonia, Spain). International Journal of Food Microbiology, 219, 56-63. doi.org/10.1016/j.ijfoodmicro.2015.12.002

Radovanovic, A., Jovancicevic, B., Arsic, B., Radovanovic, B., \& Bukarica, L. G. (2016). Application of nonsupervised pattern recognition techniques to classify Cabernet Sauvignon wines from the Balkan region based on individual phenolic compounds. Journal of Food Composition and Analysis, 49, 42-48. doi.org/10.1016/j.jfca.2016.04.001

Rinaldi, A., Blaiotta, G., Aponte, M., \& Moio, L. (2016). Effect of yeast strain and some nutritional factors on tannin composition and potential astringency of model wines. Food Microbiology, 53, 128-134. doi.org/10.1016/j.fm.2015.09.013

Rivas, G. A., Semorile, L., \& Delfederico, L. (2022). Microbial diversity of the soil, rhizosphere and wine from an emerging wine-producing region of Argentina. LWT, 153, 112429. doi.org/10.1016/j.lwt.2021.112429

Santamaría, P., Garijo, P., López, R., Tenorio, C., \& Gutiérrez, A. R. (2005). Analysis of yeast population during spontaneous alcoholic fermentation: effect of the age of the cellar and the practice of inoculation. International Journal of Food Microbiology, 103(1), 49-56. doi.org/10.1016/j.ijfoodmicro.2004.11.024
Statista. (2020). Outlook: worldwide wine. https://www.statista.com/outlook/10030000/100/win e/worldwide.

Suzzi, G., Arfelli, G., Schirone, M., Corsetti, A., Perpetuini, G., \& Tofalo, R. (2012). Effect of grape indigenous Saccharomyces cerevisiae strains on Montepulciano d'Abruzzo red wine quality. Food Research International, 46(1), 22-29. doi.org/10.1016/j.foodres.2011.10.046

Tzanetakis, N. (2006). Selection of indigenous Saccharomyces cerevisiae strains according to their oenological characteristics and vinification results. Food Microbiology, 23, 205-211. doi.org/10.1016/j.fm.2005.03.004

Van Leeuwen, C., \& Seguin, G. (2006). The concept of terroir in viticulture. Journal of Wine Research, 17(1), 1-10. doi.org/10.1080/09571260600633135

Wei, Y. J., Wu, Y., Yan, Y. Z., Zou, W., Xue, J., Ma, W. R., .. \& Wang, L. Y. (2018). High-throughput sequencing of microbial community diversity in soil, grapes, leaves, grape juice and wine of grapevine from China. PloS One, 13(3), e0193097. doi.org/10.1371/journal.pone.0193097

Zarraonaindia, I., Owens, S. M., Weisenhorn, P., West, K., Hampton-Marcell, J., Lax, S., ... \& Gilbert, J. A. (2015). The soil microbiome influences grapevineassociated Microbiota. Microbiology, 6(2), e0252714. doi.org/10.1128/mBio.02527-14

Zhang, S., Chen, X., Zhong, Q., Huang, Z., \& Bai, Z. (2017). Relations among epiphytic microbial communities from soil, leaves and grapes of the grapevine. Frontiers in Life Science, 10(1), 73-83. doi.org/10.1080/21553769.2017.1365776 